\title{
MELHORAMENTO DAS PROPRIEDADES DE PAPÉIS RECICLADOS ATRAVÉS DA ULTRASSONIFICAÇÃO DAS FIBRAS E ADIÇÃO DE XILANAS ${ }^{1}$
}

\author{
Mauro Manfredi², Rubens Chaves de Oliveira ${ }^{3}$ e Juliana Cristina da Silva ${ }^{2}$
}

\begin{abstract}
RESUMO - Nesse estudo foi avaliado o potencial de utilização de vibrações ultrassônicas e adição de xilanas para melhorar a qualidade do papel reciclado. Inicialmente, foi realizado um estudo de otimização do tratamento ultrassônico. Foram avaliadas as influências do $\mathrm{pH}$, da consistência da massa e do tempo de tratamento. A consistência da massa não afetou significativamente os resultados. Entretanto, elevados tempos de tratamento e valores de $\mathrm{pH}$ intensificaram os efeitos da ultrassonificação. O tratamento ultrassônico melhorou as propriedades mecânicas do papel de forma semelhante ao refino em PFI, embora com menor intensidade. As ondas ultrassônicas atuam, principalmente, promovendo o desfibrilamento externo das fibras. Também foram investigados os impactos da adição de xilanas na polpa. Os resultados indicaram que, mesmo com pequenas dosagens de xilanas $(10 \mathrm{~g} / \mathrm{kg})$, foi possível aumentar significativamente a resistência mecânica do papel. Dosagens acima de $50 \mathrm{~g} / \mathrm{kg}$ não são justificáveis. As tecnologias de ultrassonificação e adição de xilanas evidenciaram grande potencial técnico para melhorar a qualidade do papel reciclado.
\end{abstract}

Palavras-chave: Fibras secundárias, Ultrassom e Xilanas.

\section{DEVELOPMENT OF RECYCLED PAPER PROPERTIES BY ULTRASONIC TREATMENT AND ADDITION OF XYLAN}

\begin{abstract}
This study evaluates the potential of ultrasonic vibration application and xylan addition to improve the quality of recycled papers. Firstly, a study was conducted to optimize the ultrasonic treatment conditions. The influence of $\mathrm{pH}$, pulp consistency and treatment time were evaluated. Pulp consistency did not affect the results. On the other hand, high treatment time and $\mathrm{pH}$ intensify the ultrasonic effects. The ultrasonic vibration improved the mechanical properties of paper as a similar manner to the refine in PFI, with less intensity, however. Ultrasonic vibrations act mainly by promoting the external defibrillations of fibres. Also the effects of hemicelluloses addition were evaluated. The results showed that even with low dosage of hemicelluloses $(10 \mathrm{~g} / \mathrm{kg})$ it was possible to increase the paper strength. Dosages higher than $5 \mathrm{~g} / \mathrm{kg}$ are unwanted. The ultrasonic treatment and the xylan addition are technically efficient to improve the paper quality.

Keywords: Secondary fibers, Ultrasonic and Xylan.
\end{abstract}

\section{INTRODUÇÃO}

Durante o processo reciclagem, as fibras sofrem modificações estruturais que reduzem sua habilidade de formação da rede fibrosa. Tais modificações resultam em maior dificuldade de hidratação da parede, menor grau de colapsamento, menor ocorrência de desfibrilamento externo e menor resistência intrínseca das fibras.

Com a capacidade de hidratação reduzida, as fibras se apresentam mais resistentes ao colapsamento. Quando colapsadas, as fibras se tornam estruturas mais flexíveis, conferindo-lhes habilidade de se conformarem melhor

\footnotetext{
${ }^{1}$ Recebido em 23.09.2011 e aceito para publicação em 28.05.2012.

2 Programa de Pós-Graduação em Doutorado em andamento em Ciência Florestal. Universidade Federal de Viçosa, Depto de Engenharia Florestal - UFV, Brasil. E-mail: <mauro.manfredi@ufv.br>e < jcsfloresta@yahoo.com.br>.

${ }^{3}$ Universidade Federal de Viçosa, Centro de Ciências Agrárias, Departamento de Engenharia Florestal. Laboratório de Celulose e Papel. E-mail: <rchaves@ufv.br>.
} 
umas sobre as outras durante a formação do papel. O aumento da área de contato entre as fibras, assim como o desfibrilamento externo, favorece a formação de ligações interfibras, elevando, assim, a resistência mecânica do papel.

Uma forma de se recuperar a habilidade de formação da rede fibrosa das fibras secundárias é através do refino. No entanto, as fibras secundárias muitas vezes já sofreram refinos anteriores, o que diminui a intensidade necessária do tratamento e as torna mais suscetíveis aos efeitos negativos da operação. Mesmo quando realizado em baixas intensidades, o refino das aparas pode causar danos às estruturas das fibras (LUMIAINEN, 1992; HOLIK, 2000) e elevada produção de finos (LUMIAINEN, 1992; MINOR et al., 1993). Uma forma de contornar esses problemas seria desenvolver uma tecnologia capaz de promover o colapsamento e ativar a superfície das fibras, gerando menos danos à sua estrutura.

O uso de vibrações ultrassônicas para melhorar as propriedades mecânicas do papel já teve sua eficácia comprovada por diversos estudos envolvendo fibras virgens (LAINE et al., 1977; LAINE; GORING, 1977; WON; LEE, 1996; TATSUMI et al., 2000; PEIXOTO, 2009; BRODEUR; GERHARDSTERIN, 2010). Alguns estudos demonstraram que a ultrassonificação melhora as propriedades de forma similar ao refino mecânico, mas gera menos danos estruturais às fibras (LAINE et al., 1977; LAINE; GORING, 1977; PEIXOTO, 2009; BRODEUR; GERHARDSTERIN, 2010). O ultrassom promove o desfibrilamento externo das fibras (LAINE et al., 1977; LAINE; GORING, 1977; TATSUMI, 2000; PEIXOTO, 2009), o que aumenta o número de ligações interfibras e também o desfibrilamento interno (LAINE; GORING, 1977; TATSUMI et al., 2000; PEIXOTO, 2009), facilitando a hidratação das fibras.

Comparado ao refino convencional, o tratamento ultrassônico afeta menos o volume específico aparente para uma mesma melhoria na propriedade (LAINE et al., 1977; LAINE; GORING, 1977; WON; LEE, 1996; PEIXOTO, 2009). Tal fato sugere que o ultrassom atua, principalmente, ativando a superfície das fibras, através do desfibrilamento externo.

A ultrassonificação da polpa pode ser realizada de maneira relativamente rápida em diversos pontos do processo, como tanques e tubulações. Pode ser realizada em conjunto com o refino convencional ou, até mesmo, substituí-lo. É uma tecnologia que necessita de equipamentos pouco volumosos e permite ajustes operacionais, tornando a operação flexível quanto ao tipo de material a ser tratado. Com a ultrassonificação é possível controlar as modificações nas fibras, através da manipulação da frequência e potência das ondas (BRODEUR; GERHARDSTERIN, 2010).

Substituir o refino convencional pelo tratamento ultrassônico pode ser bastante interessante, porque permitiria alterar as propriedades do papel de forma mais seletiva, minimizando alguns efeitos negativos do refino convencional. Dessa forma, a ativação de segmentos de fibras secundárias, através da ultrassonificação, pode resultar em melhores condições operacionais e qualidade do produto final.

Outra tecnologia que pode ser utilizada para desenvolver as propriedades do papel é a adição de xilanas. O teor de xilanas afeta diretamente as propriedades do papel (ANNERGREN et al., 1962; SPIEGELBERG, 1966; SIHTOLA; BLOMBERG, 1975; MILANEZ et al., 1982; SCHÖNBERG et al., 2001; MOLIN; TEDER, 2002; SJÖBERG et al., 2002; ANJOS et al., 2005, KÖNHKE et al., 2008; MOLINA et al., 2008).

Quando presentes na superfície das fibras, as xilanas têm importante papel na formação de ligações interfibras. Quando presentes na parede, as xilanas contribuem para a hidratação, facilitando o colapsamento. Por favorecer a hidratação da parede, as xilanas tornam as fibras mais suscetíveis a ações mecânicas, reduzindo, assim, a intensidade de refino necessária para alcançar os objetivos da operação.

Diversos estudos foram realizados visando adicionar xilanas à polpa (YLLNER; ENSTRÖM, 1956; YLLNER; ENSTRÖM, 1957; AURELL, 1965; HANSSON; HARTLER, 1969; LINDER et al., 2003; KÖHNKE et al., 2008). De maneira geral, a adsorção de xilanas é favorecida pelo uso de altas temperaturas e elevados valores de pH e de tempo de tratamento. No entanto, é possível adicionar xilanas à polpa utilizando condições típicas dos processos industriais.

O objetivo deste trabalho foi melhorar as propriedades de resistência mecânica do papel reciclado, através do tratamento ultrassônico de suas fibras secundárias e através da adição de xilanas. Com este estudo, espera-se apresentar a aplicação de duas novas tecnologias que permitam produzir 
papel reciclado de melhor qualidade e, com isso, contribuir para o desenvolvimento da indústria de fibras secundárias.

\section{MATERIAL E MÉTODOS}

\subsection{Material}

Foi utilizado papel do tipo Old Corrugated Container (OCC), com comprimento médio de fibras de $0,85 \mathrm{~mm}$, coarseness de 7,15 mg/100 $\mathrm{m}$ e teor de finos de $23,8 \%$. O OCC foi mantido em água destilada por $12 \mathrm{~h}$, para posterior desagregação em desagregador laboratorial. O material foi centrifugado e armazenado em sacos plásticos em ambiente refrigerado.

Para os testes com a adição de xilanas, utilizouse um licor rico em xilanas ( $15 \mathrm{~g} / \mathrm{L})$, obtido através do processo Cold Caustic Extraction (CCE) em polpa kraft não branqueada de eucalipto. O equivalente a $300 \mathrm{~g}$ secos de polpa foi tratado com $240 \mathrm{~g}$ de $\mathrm{NaOH}$ por 30 min, em temperatura de $25^{\circ} \mathrm{C}$ e consistência de $10 \%$.

\subsection{Tratamento ultrassônico}

Inicialmente, a polpa foi submetida a 30.000 revoluções em um desagregador laboratorial com consistência de $0,8 \%$. Em seguida, a consistência da polpa foi corrigida para o valor de cada tratamento e ultrassonificada. Utilizou-se o aparelho Virsonic 475 ajustado para operar na potência de $190 \mathrm{~W}$ e frequência de ondas de $20 \mathrm{kHz}$. A ultrassonificação foi realizada em béquer contendo $1 \mathrm{~L}$ de suspensão fibrosa, mantida em movimento através de agitador magnético. Todos os tratamentos foram realizados com a temperatura inicial da suspensão em $30{ }^{\circ} \mathrm{C}$. As condições de ultrassonificação avaliadas foram a consistência de massa $(0,5 \%, 1 \%, 2 \%$ e $4 \%), \mathrm{pH}(7$ e 10$)$ e tempos de tratamento $(5,10$ e $20 \mathrm{~min})$, em esquema fatorial.

\subsection{Refino em PFI}

Amostras com massas equivalentes a $30 \mathrm{~g}$ de polpa foram desagregadas com 30.000 revoluções, num desagregador laboratorial, a uma consistência de $0,8 \%$. A massa foi, então, drenada até a consistência de $10 \%$ e submetida ao refino em moinho PFI, seguindo a norma TAPPI 248 sp-08. Após o refino, a massa foi desagregada com 10.000 revoluções. Os números de revoluções em PFI (200, 400 e 600) foram selecionados com o objetivo de obter índices de tração semelhantes aos alcançados pelo tratamento ultrassônico.

\subsection{Adição de xilanas}

O licor proveniente do CCE teve seu teor de xilanas quantificado através de espectroscopia. O licor foi armazenado em ambiente refrigerado sem a correção de $\mathrm{pH}$, que se encontrava em 13,7.

Nessa parte do experimento foram utilizadas a polpa não refinada, a polpa refinada em PFI (200 revoluções) e a polpa ultrassonificada ( 20 min em pH 8 e consistência de massa de $3 \%$ ). A adição de xilanas foi feita após o refino ou o tratamento ultrassônico. A adição se deu através da mistura do licor proveniente do CCE com a suspensão fibrosa em béquer. $\mathrm{O}$ tratamento foi realizado durante $60 \mathrm{~min}$, com temperatura de $60{ }^{\circ} \mathrm{C}$ e pH 8 . Trabalhou-se com consistência de massa de 3\%, sempre mantida sob leve agitação. As dosagens de xilanas testadas foram de $1 \%, 2,5 \%, 5 \%$ e $10 \%$ base massa seca de polpa.

\subsection{Testes físicos e mecânicos}

Foram formadas folhas com $120 \mathrm{~g} / \mathrm{m}^{2}$ para o teste de compressão e de $60 \mathrm{~g} / \mathrm{m}^{2}$ para os demais testes, seguindo-se a norma TAPPI $205 \mathrm{sp}-95$. As folhas foram acondicionadas em ambiente climatizado com temperatura de $23 \pm 1{ }^{\circ} \mathrm{C}$ e umidade relativa do ar de $50 \pm 2 \%$, para realização dos testes. Todos os testes físico-mecânicos foram realizados seguindo-se normas da TAPPI.

\subsection{Análise do material fibroso}

Foram preparadas suspensões fibrosas na consistência de $0,001 \%$, com água destilada e $2 \%$ (base polpa) de agente dispersante. As suspensões foram mantidas em repouso por um período mínimo de $4 \mathrm{~h}$, para hidratação do material fibroso.

A análise do material fibroso consistiu na mensuração do comprimento médio ponderado, coarseness e teor de finos. Foi considerado como "finos" todo elemento com comprimento inferior a 0,07 $\mathrm{mm}$. A análise foi realizada no equipamento Galai CIS-100 e processada no software-Wshape. Para o cálculo das dimensões médias das fibras, o software considerou apenas o material com comprimento entre $0,07 \mathrm{~mm}$ e $3,0 \mathrm{~mm}$.

\subsection{Análises químicas}

As análises químicas consistiram de análises de carboidratos da polpa e foram realizadas em equipamento High Performance Liquide Chromatography (HPLC).

Revista Árvore, Viçosa-MG, v.36, n.4, p.777-785, 2012 
Realizou-se uma hidrólise ácida, em que os polímeros foram convertidos em monômeros quantificáveis. $\mathrm{O}$ hidrolisado foi, então, filtrado e diluído para a concentração de leitura.

\subsection{Análise estatística}

Empregou-se um teste de identidade de modelos com teste $\mathrm{F}$ a $95 \%$ de confiança. Os modelos foram obtidos com o auxílio do software Curve Expert 1.4.

\section{RESULTADOS}

\subsection{Tratamento ultrassônico}

Os resultados evidenciaram que o tratamento das fibras com vibrações ultrassônicas permite melhorar as propriedades físico-mecânicas do papel, assim como reportado na literatura (LAINE et al., 1977; LAINE; GORING, 1977; WON e LEE, 1996; TATSUMI et al., 2000; PEIXOTO, 2009; BRODEUR; GERHARDSTERIN, 2010).

A Figura 1 mostra o efeito do tratamento ultrassônico quando realizado em diferentes condições de $\mathrm{pH}$, tempo e consistência de massa. De acordo com o teste de identidade de modelo com $95 \%$ de confiança, não houve diferença significativa entre os resultados das diferentes consistências testadas. Com isso, todas as consistências foram representadas por apenas uma curva gerada por uma equação comum.

O ultrassom promoveu incrementos de aproximadamente $35 \%$ e $40 \%$ no índice de tração, quando realizado em pH 7 e pH 10, respectivamente. Na condição mais alcalina, o efeito da ultrassonificação apareceu mais rapidamente, atingindo o máximo de incremento com apenas 10 min de tratamento (Figura 1A).

Com o tratamento ultrassônico, o volume específico aparente (VEA) do papel decresceu 4\%, independentemente do $\mathrm{pH}$ do meio (Figura 1B).

Para comparar a tecnologia de ultrassom com uma tecnologia de refino convencional, foram realizados refinos em moinho PFI com até 600 revoluções e comparado com os tratamentos ultrassônico em pH 7 , com tempos de até $20 \mathrm{~min}$. A Figura 2 compara os efeitos dessas duas tecnologias, tomando como base comparativa o VEA. Os resultados demonstraram que o tratamento ultrassônico não foi tão eficiente quanto ao refino em PFI para desenvolver resistência mecânica do papel.

Na Figura 2 é possível notar que, para um mesmo valor de VEA, o tratamento com ultrassom produz polpa com maior índice de tração e maior grau Schopper Riegler ( $\left.{ }^{\circ} \mathrm{SR}\right)$. No entanto, o maior valor de índice de tração foi obtido pelo refino em PFI.

\subsection{Adição de xilanas}

Os resultados indicaram que apenas parte das xilanas adicionadas foi retida na polpa (Tabela 1). Em baixas dosagens $(10 \mathrm{~g} / \mathrm{kg})$, a retenção foi de $100 \%$ nas polpas refinadas ou ultrassonificadas, mas de apenas $70 \%$ na polpa original. As taxas de retenção decresceram significativamente com o aumento da dosagem, tendendo
A Índice de tração

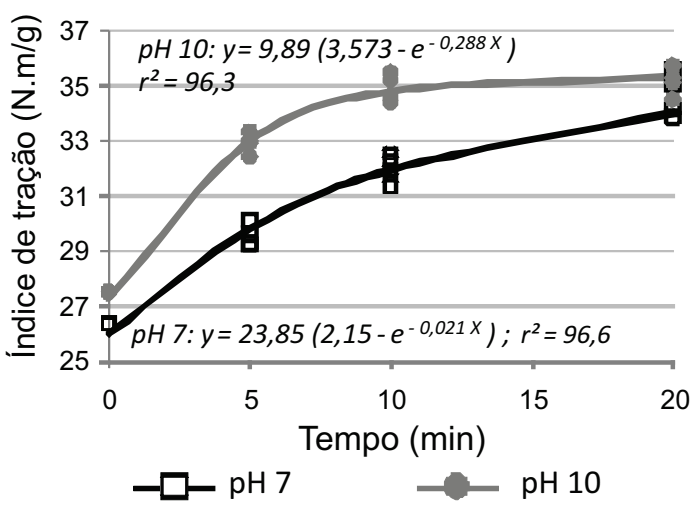

B Volume Específico Aparente

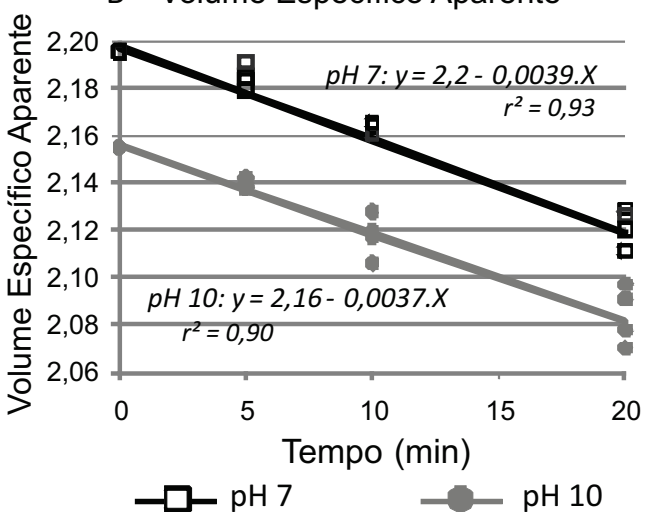

Figura 1 - Efeito do tratamento ultrassônico no índice de tração (A) e no VEA (B).

Figure 1 -Effect of the ultrasonic treatment in the tensile index (A) and bulk (B).

Revista Árvore, Viçosa-MG, v.36, n.4, p.777-785, 2012 
A

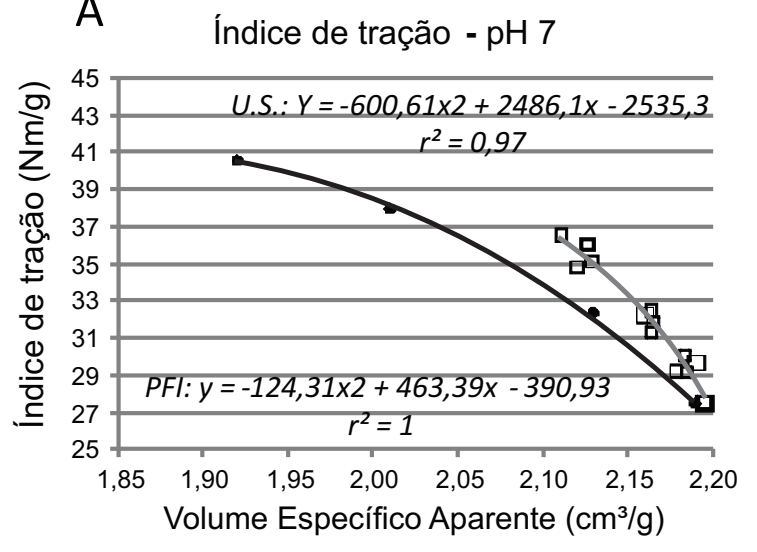

B

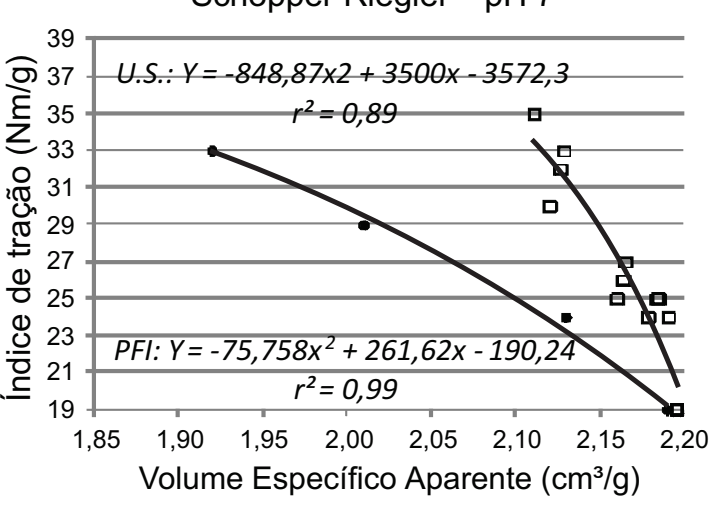

$\mathrm{PFI}$

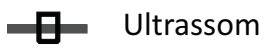

Figura 2 - Comparação entre efeitos do ultrassom e do PFI nas propriedades da polpa.

Figure 2 - Comparison between the ultrasound and PFI in the pulp properties.

a uma estabilização. As menores taxas de retenção foram de $24 \%$ para polpa original e de $50 \%$ para as polpas tratadas com ultrassom ou refinadas em PFI.

Quando foram aplicadas altas dosagens, observouse que a taxa de retenção (em percentual) pouco foi alterada. Passando da dosagem de $50 \mathrm{~g} / \mathrm{kg}$ para 100 $\mathrm{g} / \mathrm{kg}$, a taxa de retenção permaneceu praticamente a mesma. Esse fenômeno foi observado tanto no caso da polpa-referência quanto no caso das polpas refinadas em PFI ou ultrassonificadas.

A Figura 3 apresenta o efeito da adição de xilanas na resistência à tração do papel. O melhoramento máximo

Tabela 1 - Adsorção de xilanas em polpa original, refinada e ultrassonificada.

Table 1 - Retention of xylans in original, refined and ultrasonificated pulps.

\begin{tabular}{lcc}
\hline Polpa & Dosagem $(\mathrm{g} / \mathrm{kg}$ polpa $)$ & Retenção $(\%)$ \\
\hline Original & 10 & 70 \\
& 25 & 36 \\
& 50 & 30 \\
Refinada em PFI & 100 & 24 \\
& 10 & 100 \\
& 25 & 80 \\
Ultrassonificada & 50 & 52 \\
& 100 & 52 \\
& 10 & 100 \\
& 25 & 92 \\
& 50 & 60 \\
\hline
\end{tabular}

dessa propriedade foi obtido com a dosagem máxima de $100 \mathrm{~g} / \mathrm{kg}$. Nessa condição, foi observado incremento do índice de tração de aproximadamente $37 \%$ na polpa original, $43 \%$ na polpa refinada em PFI e $36 \%$ na polpa ultrassonificada. No entanto, mesmo com a dosagem de $10 \mathrm{~g} / \mathrm{kg}$ houve expressivo incremento do índice de tração.

Os resultados apontaram que há pouco efeito na propriedade quando se eleva a dosagem de $5 \%$ para $10 \%$. Também foi possível constatar que a polpa refinada em PFI apresentou os maiores índices de tração, enquanto menores índices foram observados na polpa original.

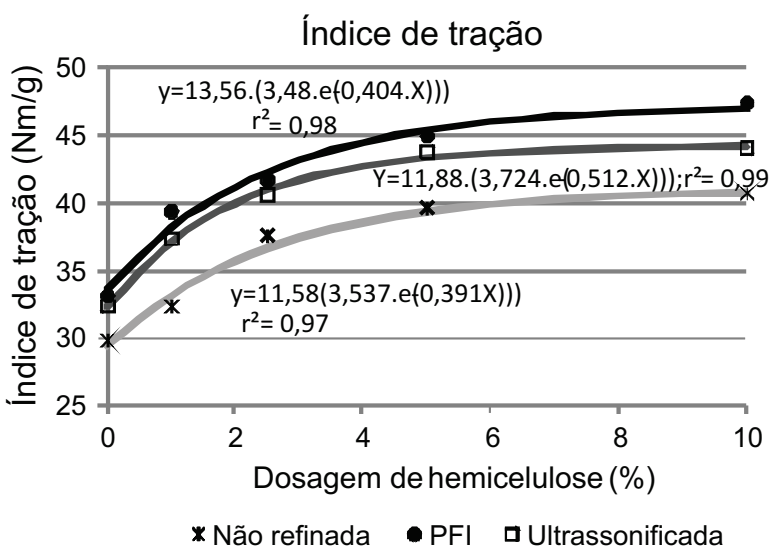

Figura 3 - Efeito da adição de xilanas na resistência à tração. Figure 3 - Effect of the xylan addition in the tensile index.

Revista Árvore, Viçosa-MG, v.36, n.4, p.777-785, 2012 


\section{DISCUSSÃO}

\subsection{Tratamento ultrassônico}

Como observado na Figura 1A, a condição alcalina do meio facilitou a ação das ondas ultrassônicas, o que está de acordo com a literatura (PEIXOTO, 2009; WON; LEE, 1996). Apesar de atingir valores finais semelhantes, o índice de tração da polpa tratada em meio alcalino necessitou de menor tempo de tratamento para responder, positivamente, ao tratamento. O índice de tração é fortemente afetado pela capacidade de ligações interfibras. O número de ligações interfibras depende, principalmente, do tamanho da área de contato entre as fibras e das características da superfície da fibra.

A área de contato interfibra eleva-se com o aumento da flexibilidade das fibras, que é obtido, principalmente, através da hidratação e colapsamento da parede. O número de sítios de ligações por unidade de área superficial das fibras também afeta a formação de ligações interfibras. É possível aumentar esse número de sítios de ligações promovendo o desfibrilamento externo das fibras. Com isso, o desenvolvimento mais acentuado do índice de tração, quando em pH 10, sugere que a alcalinidade do meio intensificou o colapsamento e, ou, a ação de desfibrilamento externo causados pelo ultrassom. No entanto, ao avaliar os efeitos do tratamento no VEA do papel, foi observado que o $\mathrm{pH}$ do meio não influenciou na ação do ultrassom (Figura 1B).

A Figura 1B mostra que o comportamento da propriedade ao longo do tratamento foi o mesmo nos dois valores de $\mathrm{pH}$. A diferença nos valores de VEA entre os tratamentos em pH 7 e pH 10 não se deve ao efeito do ultrassom e, sim, ao efeito isolado do pH no grau de hidratação das fibras. Isso pode ser constatado observando os valores de VEA das polpas não ultrassonificadas (tempo zero) em pH 7 e pH 10. Em meio alcalino, o VEA foi menor do que no $\mathrm{pH} 7$, e tal diferença se manteve constante conforme se promoveu a ultrassonificação, o que significa que o ultrassom atuou com a mesma intensidade nas duas condições de $\mathrm{pH}$.

O VEA depende, principalmente, do grau de colapsamento das fibras. O fato de o tratamento ultrassônico pouco ter afetado o VEA, mas ter promovido aumento do índice de tração, sugere que o ultrassom age preferencialmente promovendo o desfibrilamento externo das fibras, com pouco impacto no colapsamento das fibras. Além do mais, a Figura 1B mostra que a ação das ondas ultrassônicas não foi afetada pelo $\mathrm{pH}$, diferentemente do que se observou com relação ao índice de tração (Figura 1A). Tais comparações evidenciam que a elevação do $\mathrm{pH}$ potencializa, principalmente, a ação das ondas ultrassônicas em promover o desfibrilamento externo e não em colapsar as fibras.

No meio alcalino, as superfícies das fibras se encontram mais abertas, com maiores diâmetros dos poros. Com isso, intensifica-se o surgimento de cavitação nas áreas da superfície das fibras, resultando em desfibrilamento externo mais pronunciado. Dessa forma, quanto maior o $\mathrm{pH}$, menor o tempo de tratamento necessário para se obter determinado valor para uma propriedade.

\subsection{Ultrassom $v s$. refino mecânico}

Os resultados demonstraram que para um mesmo VEA o índice de tração da polpa ultrassonificada foi maior do que o da polpa refinada em PFI. Como o VEA está fortemente associado ao colapsamento das fibras, constatou-se que, comparado com o PFI, o ultrassom aumenta o número de ligações interfibras através de um mecanismo menos dependente do colapsamento das fibras. Um possível mecanismo é o desfibrilamento externo, que aumenta a superfície específica das fibras e produz finos. Estes preenchem os espaços interfibrilares, aumentando o contato interfibra e, consequentemente, o número de ligações interfibras.

A Figura 2B demonstra que para um mesmo VEA a polpa ultrassonificada apresentou maior ${ }^{\circ} \mathrm{SR}$. Assim como o VEA, o ${ }^{\circ} \mathrm{SR}$ é fortemente afetado pelo grau de colapsamento das fibras e pelo teor de finos. Portanto, tal constatação indica que para um mesmo ${ }^{\circ} \mathrm{SR}$ a polpa ultrassonificada deve apresentar menor grau de colapsamento das fibras e maior teor de finos do que a polpa refinada em PFI. Tal consideração novamente aponta para a hipótese de que o ultrassom atua, principalmente, através do desfibrilamento externo. Uma análise do teor de finos foi realizada, cujos resultados confirmaram o esperado (Figura 4).

Os resultados da Figura 4 confirmaram a hipótese levantada sobre o mecanismo de ação do tratamento ultrassônico. A ultrassonificação não é tão eficiente quanto o refino em PFI para promover o colapsamento 


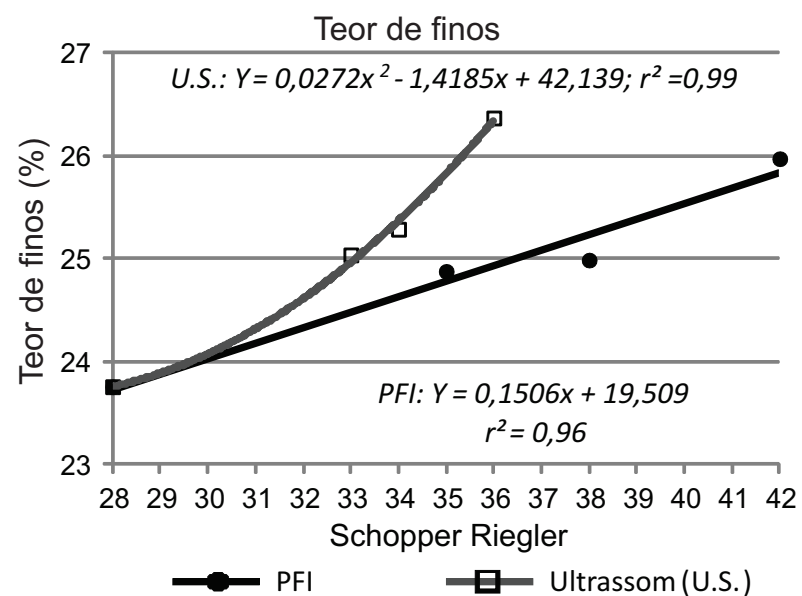

Figura 4-Teor de finos de polpas ultrassonificadas ou refinadas em PFI.

Figure 4-Fines rate of ultrasonificated or refined pulps.

das fibras. Entretanto, o tratamento ultrassônico atua, principalmente, na superfície das fibras, promovendo o desfibrilamento externo, desprendendo materiais das fibras e elevando o teor de finos da massa (LAINE et al., 1977; LAINE; GORING, 1977; WON; LEE, 1996; TATSUMI et al., 2000; PEIXOTO, 2009).

\subsection{Adição de xilanas}

Os resultados demonstraram que a polpa refinada em PFI e a polpa ultrassonificada retiveram mais xilanas do que a polpa original. Quando submetida a um desses tratamentos, a superfície externa das fibras se encontrava mais desfibrilada, o que aumentou o número de sítios de ligação aptos a adsorver as xilanas. Além do mais, tais polpas tendem a reter maior quantidade de xilanas durante a drenagem da massa. Devido à maior flexibilidade e teor de finos, as polpas refinadas ou ultrassonificadas possuem drenagem mais lenta. Essa menor velocidade do fluxo de água pela rede fibrosa facilita a retenção das unidades de xilanas.

O decréscimo na taxa de retenção com o aumento da dosagem pode ser explicado pela disponibilidade de sítios de ligação aptos para adsorver as xilanas. Quando são aplicadas pequenas dosagens, as cadeias de xilanas se conectam facilmente com esses sítios devido à disponibilidade destes. Com o aumento da dosagem, as xilanas encontram maior dificuldade para se ligarem às fibras, em razão da menor disponibilidade de sítios de ligação.
Quando se aplicam dosagens bastante elevadas, observa-se que a taxa de retenção pouco se altera. Passando da dosagem de $50 \mathrm{~g} / \mathrm{kg}$ para $100 \mathrm{~g} / \mathrm{kg}$, a taxa de retenção permaneceu praticamente a mesma. Isso significa que aproximadamente o dobro de xilanas foi retido no papel. Uma possível explicação é que, conforme aumenta a concentração de xilanas, ocorre a formação de agregados de xilanas (LINDER et al., 2003). A formação de agregados favorece a adsorção das xilanas nas fibras (HENRIKSSON; GATENHOLM, 2001; HANNUKSELA et al., 2002; LINDER et al., 2003; KABEL et al., 2006; KÖHNKE et al., 2008).

Conforme reportado por outros pesquisadores, as xilanas contribuíram significativamente para a resistência mecânica do papel (SCHÖNBERG et al., 2001; ANJOS et al., 2005; DANIELSSON; LINDSTRÖM, 2005; KÖNHKE; GATENHOLM, 2007; SHIN; STROMBERG, 2007; KÖNHKE et al., 2008; MOLINA et al., 2008). Os resultados apresentados na Figura 3 demonstraram que, mesmo com pequenas dosagens, houve expressivo incremento do índice de tração e que, a partir da dosagem de $50 \mathrm{~g} / \mathrm{kg}$, pouco efeito foi observado.

As xilanas têm importante papel na formação de ligações interfibras e hidratação da parede. Apesar de o teor de xilanas do papel continuar aumentando, a partir de determinadas dosagens os efeitos no índice de tração são pequenos. Uma possível explicação para isso está no fato de as xilanas se agruparem em agregados. Quando em agregados, as xilanas realizam menor número de ligações interfibras, uma vez que não se encontram dispersas nas superfícies das fibras.

A polpa refinada em PFI apresentou os maiores índices de tração, seguida da polpa ultrassonificada e da polpa original. Tal fato se deve ao tratamento que cada polpa recebeu antes da aplicação de xilanas, o que é constatado pelos seus valores iniciais de índice de tração. Conforme se adicionou às xilanas, as polpas apresentaram comportamento das curvas de índice de tração semelhantes entre si devido à ocorrência da mesma redução na taxa de retenção de xilanas. Ou seja, nas três polpas, as maiores taxas de retenção foram observadas em baixas dosagens. Conforme aumentou a dose de xilanas, observou-se semelhante redução na taxa de retenção de xilanas das três polpas.

Revista Árvore, Viçosa-MG, v.36, n.4, p.777-785, 2012 


\section{CONCLUSÕES}

A ultrassonificação da polpa resultou em melhorias nas propriedades mecânicas do papel reciclado. Em meio alcalino, os efeitos surgiram com menor tempo de tratamento. As propriedades do papel foram melhoradas com pouco impacto no volume específico aparente do papel.

Adicionar xilanas ao papel aumentou significativamente a sua resistência à tração. A retenção de xilanas foi favorecida por um tratamento prévio da polpa em moinho PFI ou com o ultrassom. Doses de xilanas acima de $50 \mathrm{~g} / \mathrm{kg}$ de polpa não são justificáveis.

As tecnologias de tratamento ultrassônico e a adição de xilanas comprovaram a sua eficácia técnica para melhorar a qualidade do papel reciclado.

\section{AGRADECIMENTOS}

Ao Conselho Nacional de Desenvolvimento Científico e Tecnológico, pelo apoio.

\section{REFERÊNCIAS}

ANJOS, O.; SANTOS, A.; SIMÕES, R. Efeito do teor de xilanas na qualidade do papel produzido com fibra de Eucalipto. In: CONGRESSO FLORESTAL NACIONAL, 5., 2005, Viseu. Anais... Lisboa: SPCF, 2005.

ANNERGREN, G.; RYDHOLM, S.; VARDHEIM, S. Influence of raw material and pulping process on the chemical composition and physical properties of paper pulps. Svensk Papperstidning, v.66, n.6, p.196-210, 1962.

AURELL, R. Increasing kraft pulp yield by redeposition of hemicelluloses. Tappi Journal, v.2, n.48, p.80-84, 1965.

BRODEUR, P. H.; GERHARDSTEIN, J. P. Overview of applications of ultrasonics in pulp and paper industry. In: ULTRASONICS SYMPOSIUM, 1998, Senday. Disponível em: <www.ieexplore.ieee.org $>$. Acesso em: 23 de fev. 2010.

DANIELSSON, S.; LINDSTRÖM, M. E. Influence of birch xylan adsorption during kraft cooking on softwood pulp strength.

Nordic Pulp \& Paper Research Journal, v.20, n.4, p.436-441, 2005.
HANNUKSELA, T.; TENKANEN, M.; HOLMBOM, B. Sorption of dissolved galactoglucomannans and galactomannans to bleached kraft pulp. Cellulose, v.9, n.3-4, p.251-261, 2002.

HANSSON, J. A.; HARTLER, N. Sorption of hemicelluloses on cellulose fibers. Svensk Papperstidning, v.17, n.72, p.521-530, 1969.

HENRIKSSON, A.; GATENHOLM, P.

Controlled assembly of glucuronoxylans onto cellulose fibres. Holzforschung, v.55, n.5, p.494-502, 2001.

HOLIK, H. Unit operations and equipment in recycled fiber processing. In: GÖTTSCHING, L.; PAKARINEN, H. Recycled fiber and deinking. Helsinki: PI/Tappy Press, 2000. p.91-209.

KABEL, M. A. et al. Structural differences of xylans affect their interaction with cellulose. Carbohydrate Polymers, v.69, n.1, p.94-105, 2006.

KÖHNKE, T.; GATENHOLM, P. The effect of controlled glucouronoxylan adsorption on dryinginduced strength loss of bleached softwood pulp. Nordic Pulp \& Paper Research Journal, v.22, n.4, p.508-515, 2007.

KÖHNKE, T. et al. The effect of barley husk arabinoxylan adsorption on the properties of cellulose fibres. Cellulose, v.15, n.14, p.537-546, 2008.

LAINE, J. E.; GORING, D. A. I. Influence of ultrasonic irradiation on the properties of cellulosic fibres. Cellulose Chemistry and Technology, v.11, n.5, p.561-567, 1977.

LAINE, J. E. et al. Application of ultrasound in pulp and paper technology. Paper Ja Puu: Paper och Trä, v.59, p.235-247, 1977.

LINDER, A. et al. Mechanism of assembly of xylan onto cellulose surfaces. Langmuir, v.19, n.12, p.5072-5077, 2003.

LUMIAINEN, J. J. Refining recycled fibers: advantages and disadvantages. Tappi Journal, v.75, n.8, p.92-97, 1992. 
MILANEZ, A. F. et al. Influência das xilanass nas propriedades óticas e físico-mecânicas da polpa. In: CONGRESSO E EXPOSIÇÃO ANUAL DE CELULOSE E PAPEL ABTCP, 15., 1982, São Paulo. Anais... São Paulo: ABTCP, 1982. p.155-170.

MINOR, J. L.; SCOTT, C. T.; ATALLA, R. H. Restoring bonding strength to recycled fiber. In. RECYCLING SYMPOSIUM, 1993, New Orelans. Anais... Atlanta: Tappi Press, 1993. p.379-381.

MOLIN, U.; TEDER, A. Importance of cellulose/ hemicellulose ratio for pulp strength. Nordic Pulp Paper Research Journal, v.17, n.1, p.14, 2002.

MOLINA, E. M. A.; MOGOLLÓN, G.;

COLODETTE, J. L. Efecto de las xilanas en la refinabilidad y propiedades físico-mecánicas de pulpa kraft de eucalyptus spp. In: CONGRESO IBEROAMERICANO DE INVESTIGACIÓN EN CELULOSA Y PAPEL, 2008, Guadalajara. Anais... Guadalajara: CIADICYP, 2008.

PEIXOTO, R. S. Utilização de vibrações ultra-sônicas para o refino de celulose kraft de eucalipto. 2002. 77f. Tese (Mestrado em Ciência Florestal) - Universidade Federal de Viçosa, Viçosa, MG, 2009.

SCHÖNBERG, C. et al. The importance of xylan for the strength properties of spruce kraft fibres.

Holzforschung, v.55, p.639-644, 2001.

SHIN, N.; STROMBERG, B. Xylan's impact on eucalyptus pulp yield and strength - Myth or reality? In: WORKSHOPONCHEMICAL PULPING PROCESS, 1., 2006, Karlstand. Proceedings... Karlstand: 2006.
SIHTOLA, H.; BLOMBERG, L. Hemicelluloses precipitated from steeping liquor in the viscose process as additives in papermaking.

Cellulose Chem. and Technology, v.9, n.5, p.555-560, 1975.

SJÖBERG, J. et al. Analysis of carbohydrate and lignin in the surface and inner layers of softwood pulp fibers obtained employing various alkaline cooking process. Nordic Pulp and Paper Research Journal, v.17, p.295-301, 2002.

SPIEGELBERG, H. L. The effect of hemicelluloses on the mechanical properties of individual pulp fibres, Tappi Journal, v.49, n.9, p.388, 1966 .

TATSUMI, D. et al. Ultrasonic treatment to improve the quality of recycled pulp fiber. Journal of Wood Science, v.46, n.5, p.405409. 2000.

WON, J-M.; LEE, M-K. Effect of ultrasonic treatment on the pulp properties. Journal of Korea Tappi, v.28, n.2, p.46-54, 1996.

YLLNER, S.; ENSTRÖM, B. Studies of the adsorption of xylan on cellulose fibres during the sulphate cook. Part. 1. Svensk

Papperstidning, v.59, n.6, p.229, 1956.

YLLNER, S.; ENSTRÖM, B. Studies of the adsorption of xylan on cellulose fibres during the sulphate cook. Part. 2. Svensk

Papperstidning, v. 60, n. 6, p. 449, 1957. 
